Instituto Internacional de Investigación y Desarrollo Tecnológico Educativo INDTEC, C.A.

DOI: https://doi.org/10.29394/Scientific.issn.2542-2987.2020.5.E.10.205-225

OAI-PMH: http://www.indteca.com/ojs/index.php/Revista Scientific/oai

Artículo Original / Original Article

\title{
Predisposición al consumo de sustancias en aspirantes a la carrera militar
}

Autores: Joel Santiago Barreno López Universidad Internacional SEK, UISEK jsbarreno.mpp@uisek.edu.ec

Quito, Ecuador

https://orcid.org/0000-0002-5385-5411

Claudio Danilo Pazmiño Tirado Universidad Internacional SEK, UISEK cdpazmino.mpp@uisek.edu.ec

Quito, Ecuador

https://orcid.org/0000-0002-6964-2795

Luis Alfonso Iriarte Pérez Universidad Internacional SEK, UISEK luisiriartep@gmail.com

Quito, Ecuador https://orcid.org/0000-0002-8422-7047

\section{Resumen}

El presente estudio tiene como objetivo determinar la relación entre las actitudes o maneras de pensar y los patrones de conducta frente al consumo de sustancias en aspirantes a la Carrera Militar. Comprende un estudio descriptivo y se ha utilizado la metodología cuantitativa para el análisis de poblaciones, se contó con la participación de 1118 sujetos de edades comprendidas entre los 18 y 26, con una media de edad de 20,41 años, a quienes se administró el Cuestionario de Actitudes hacia el Alcohol y Drogas llegales (CAADI) y el Autoinforme de Consumo de Sustancias Psicoactivas (AICA). Como resultados, se halló que la conciencia sobre los efectos negativos del consumo de sustancias se relaciona con el rechazo e índice de desagrado de consumo de drogas. Se encontró diferencias estadísticamente significativas entre aspirantes de primer y segundo año. Adicionalmente se identificó relación entre las actitudes y la disposición hacia las drogas, a pesar de tener la conciencia de los efectos nocivos. Por esta razón, concluimos que la conciencia de los efectos negativos sobre el consumo tiene relación significativa con la tendencia actitudinal en los aspirantes y la disposición comportamental. La identificación religiosa es un factor de protección frente al consumo de sustancias.

Palabras clave: adicción; militarismo; consumo; estupefaciente.

Cómo citar este artículo:

Barreno, J., Pazmiño, C., \& Iriarte, L. (2020). Predisposición al consumo de sustancias en aspirantes a la carrera militar. Revista Scientific, 5(Ed. Esp.), 205-225, e-ISSN: 2542-2987. Recuperado de: https://doi.org/10.29394/Scientific.issn.2542-2987.2020.5.E.10.205-225

Fecha de Recepción: 11-04-2020
Fecha de Aceptación: 19-06-2020
Fecha de Publicación: 05-08-2020 
Instituto Internacional de Investigación y Desarrollo Tecnológico Educativo INDTEC, C.A.

DOI: https://doi.org/10.29394/Scientific.issn.2542-2987.2020.5.E.10.205-225

OAI-PMH: http://www.indteca.com/ojs/index.php/Revista_Scientific/oai

Artículo Original / Original Article

\section{Predisposition to substance use in applicants to the military career}

\section{Abstract}

The objective of this study is to determine the relationship between attitudes or ways of thinking and behavior patterns regarding substance use in applicants to the Military Career. It comprises a descriptive study and the quantitative methodology has been used for the analysis of populations. 1118 subjects between the ages of 18 and 26 participated, with an average age of 20.41 years, who were administered the Questionnaire on Attitudes towards Alcohol and Illegal Drugs (CAADI) and the Self-Report on the Use of Psychoactive Substances (AICA). As results, it was found that awareness of the negative effects of substance use is related to the rejection and dislike rate of drug use. Statistically significant differences were found between first and second year applicants. Additionally, a relationship between attitudes and disposition towards drugs was identified, despite being aware of the harmful effects. For this reason, we conclude that awareness of the negative effects on consumption has a significant relationship with attitudinal tendency in applicants and behavioral disposition. Religious identification is a protective factor against substance use.

Keywords: addiction; militarism; consumption; narcotic drugs.

\footnotetext{
How to cite this article:

Barreno, J., Pazmiño, C., \& Iriarte, L. (2020). Predisposition to substance use in applicants to the military career. Revista Scientific, 5(Ed. Esp.), 205-225, e-ISSN: 2542-2987. Recovered from: https://doi.org/10.29394/Scientific.issn.2542-2987.2020.5.E.10.205-225
}

Date Received: 11-04-2020
Date Acceptance:

19-06-2020
Date Publication:

05-08-2020 


\section{Introducción}

La Organización de los Estados Americanos (2019a): en su Informe sobre el Consumo de Drogas de las Américas, presenta datos actualizados de las drogas más utilizadas dentro de la región, diferenciando por tipo de sustancias y grupo de personas. De un estimado de mil millones de personas en los 35 países, con diferencias culturales y sociales o con problemas diversos relacionados al consumo, reconocen factores comunes en la población en general. Estos factores son: la edad, el apoyo familiar.

Según el informe de la Organización de los Estados Americanos (2019b): se establecieron los siguientes aspectos de análisis: la edad promedio de consumo, diferencias por género, tendencias relacionadas al consumo y nuevos desafíos de orden político en materia de drogas.

En este mismo sentido, la Organización de los Estados Americanos (2019c): ha encontrado que el tabaco es la única sustancia que a largo plazo muestra una disminución en su consumo. De acuerdo con la Organización Mundial de la Salud, World Health Organization (2014): en América latina la cerveza es el producto con mayor prevalencia de consumo con un 53\%, seguido del whisky con el $32 \%$ y vino con el $11 \%$. De igual manera, en Ecuador la cerveza encabeza esta lista con un $67 \%$ de consumo.

La Organización de los Estados Americanos (2019d): en un análisis de exposición al consumo de tabaco, cannabis, alcohol y cocaína durante el último mes, se encontró que en El Salvador el 9,5\% de la población en edades entre los 17 y 24 años consumió alguna sustancia, mientras que en Argentina y Uruguay la cifra llegó al $52 \%$. Chile es el país que presenta el nivel más elevado del consumo de tabaco en la región con un $38,7 \%$. Señalando a la Organización Panamericana de la Salud (OPS, 2018): se ha encontrado que no existen diferencias significativas entre hombres y mujeres.

A su vez, en un estudio realizado por el Observatorio de Dogas de Colombia (2014), encontró que: el $24,1 \%$ de la población evaluada mencionó 
que había fumado tabaco alguna vez en su vida, con una prevalencia mayor en hombres que en mujeres relacionada a la permisividad familiar y cuestiones culturales de género. En esta misma línea de investigación, Arteaga (2015): indica que en Colombia, se determinó que existe una relación significativa entre el consumo de marihuana, alcohol y la cocaína.

Haciendo referencia a factores asociados al consumo de sustancias, Mendoza y Vargas (2017): mencionan que los principales factores son: la funcionalidad de la familia, el contacto con el medio social y académico. Por otro lado, existen numerosos factores que guardarían relación con el consumo: alivio de ansiedad, impulso por desvincularse de los problemas, inadecuado manejo de la frustración y una constante búsqueda de nuevas experiencias generadoras de placer.

Este aspecto concuerda con la investigación de Arrioja, Ávila, Frari, Gargantua y Morales (2017): quienes mencionan que el consumo es un problema de tipo social que afecta de manera alarmante a los jóvenes. Además, destacan factores como la edad y el género para la aparición de conductas relacionadas al consumo de alcohol.

Por su parte, en la Constitución de la República del Ecuador (2008): se reconoce que "las adiciones son un problema de salud púbica" (art. 364). En tal sentido ha implementado políticas públicas encaminadas a la prevención, promoción y atención mediante tratamientos en diferentes modalidades (ambulatorio básico, intensivo y residencial), además de la prohibición de la criminalización del consumo.

En cuanto a Vélez y Gudiño (2017): las políticas públicas relacionadas al consumo de sustancias ilícitas y la tenencia de estas, de acuerdo con la reforma establecida en la tenencia de sustancias, se ha evidenciado un incremento en el consumo de sustancias en el año 2006. Otra de las instancias legalmente constituidas es la Secretaria Técnica de Drogas, que se instauró en el año 2015, con el propósito de trabajo familiar-comunitario. En este 
sentido, Fuentes, Litardo y Castro (2017): consideran a la reducción de riesgos y daños, la inclusión social y la participación ciudadana como uno de los principales factores de trabajo.

Al respecto, una investigación realizada en Ecuador por Ruisoto, Cacho, López-Goñi, Vaca y Jiménez (2016): se encontró que existe una mayor prevalencia de consumo de alcohol en hombres en un $92,24 \%$ y en las mujeres en un 82,86\%. Al mismo tiempo, Meneses, Cisneros y Braganza (2019): en un estudio de factores sociales asociados al consumo excesivo de alcohol, encontró que las personas en condiciones de pobreza y no han culminado sus estudios o en desempleo, tienen mayor probabilidad de desarrollar un problema en cuanto al consumo de alcohol.

Para autores como Moreta-Herrera, Ilaja-Verdesoto, Mayorga-Lascano, León-Tamayo y López-Castro (2018a): la exposición de los adolescentes a drogas legales como el alcohol y el tabaco tienen un alto índice de prevalencia, mientras que en el uso de sustancias ilegales existe un predominio de consumo de cannabis. En cuanto al consumo de drogas en la vida militar, en una Escuela de Formación de Soldados del Ejército del Ecuador ha brindado las facilidades necesarias para la realización de este estudio. Institución donde se presentan niveles de consumo medios y bajos de tabaco, alcohol y otras sustancias.

Tal como lo indica Espinoza (2019a): la falta de programas de adaptación socioeducativa en el contexto de la formación militar, incide directamente en los procesos de aprendizaje, así como en el bienestar subjetivo. Respecto a investigaciones previas relacionadas con la atención de este tipo de población, podemos hacer referencia a programas y evidencias de Estados Unidos y otros países. Ante esto, el Instituto Nacional sobre el Abuso de Drogas, National Institute on Drug Abuse (2019): informa que los militares tienen efectos en su salud producto de los despliegues militares y el ritmo de vida que llevan hace que desarrollen factores de riesgo. Sin embargo, 
algunos militares pueden contar con factores de protección, tales como: las relaciones familiares, la participación en programas inclusivos de tipo comunitarios y la estabilidad dentro de esta comunidad. Por demás de tener espacios de práctica de alguna actividad deportiva y vínculos emocionales seguros.

En la encuesta realizada por Meadows, et al. (2018a): sobre los comportamientos de la salud y bienestar de los militares en Estados Unidos, se destaca que: el $67,6 \%$ de los miembros desplegados en combate, han demostrado el consumo de alguna sustancia, como alcohol en un $32 \%$ y tabaco en un $28 \%$, mientras que el $0,1 \%$ consumo de opiáceos.

Mencionando a Gribble, Goodwin y Fear (2019): el despliegue militar no sólo afecta a los oficiales que están en servicio, sino también a sus familias. Esto coincide con los resultados de la encuesta realizada por Meadows, et al. (2018b): del Departamento de Defensa de los Estados Unidos, donde se encontró que el $33,2 \%$ de los militares presentaban conductas relacionadas a un consumo peligroso. Para el contexto brasileño, da Silva, Paula y Assunção (2019): hacen referencia al uso de ansiolíticos en bomberos militares y concluyeron que el 9,8\% de los participantes en la investigación registró un consumo de ansiolíticos.

De la casuística expuesta se reconoce un alto índice de incidencia de consumo de alcohol, tabaco, ansiolíticos y benzodiacepinas en población militar, potencialmente de problemas asociados al consumo, que han tenido efectos incluso en las estructuras familiares. Esto concuerda con la investigación realizada en España por Vargas, Castellano y Trujillo (2017a): con un grupo de 605 militares desplegados en combate en Bosnia, se evidencia que el tabaco fue una de las sustancias más usadas con un $54,2 \%$ seguido del alcohol en un $39,9 \%$, esto en base a las drogas legales, en contraste a las ilegales, se registró que el $36,2 \%$ consumió alguna vez cannabis, luego estuvo la cocaína con el $14,9 \%$ y finalmente las anfetaminas 
en un $12,1 \%$, lo que indica que existe una problemática significativa de consumo en población militar. En este mismo orden de ideas, Vargas, Castellano y Trujillo (2017b): concluyeron que el apoyo social se convierte en un factor protector frente al consumo.

Por su parte, Espinoza (2019b): refiere que el consumo de drogas guarda estrecha relación con el aumento de edad. A lo que alegan MoretaHerrera, Ilaja-Verdesoto, Mayorga-Lascano, León-Tamayo y López-Castro (2018b): que existen diferencias significativas entre el género, actitudes y predisposición al consumo.

Consideramos que hablar de actitudes guarda relación con motivaciones implícitas frente al consumo, idea que concuerda con lo propuesto por Duarte, Varela, Salazar, Lema y Tamayo (2012a): ya que encontraron que el consumo se relaciona con las siguientes motivaciones: sentirse mejor y reducir la tensión de estar en lugares como el medio universitario. Tomamos en consideración a Santrock (2002): quien habla sobre la motivación, entendida como las razones de comportarse.

Mientras que para Duarte, Varela, Salazar, Lema y Tamayo (2012b): en el caso de las sustancias ilegales las motivaciones pueden variar: experimentar sensaciones diferentes y placenteras, sentirse mejor. La teoría o modelo de acción razonadora expuesta por Fishbein (1980): en la que se establece la suma de un factor de tipo social y un aspecto personal, determina las intenciones de un comportamiento. En atención a Vera, Quintana, Valdés, Martínez y Martínez (2014): la teoría razonadora nos dice que la noción del comportamiento se determina por la información que la persona tenga a su disposición frente a una manera de hablar.

El presente recorrido teórico nos ha permitido empezar a percibir cuál es el lugar que tiene el consumo de drogas en el contexto ecuatoriano y regional, y cuál es la actitud o disposición que se tiene frente a estas sustancias lícitas e ilícitas. Así, consideramos que el objetivo de la presente 
investigación es determinar la existencia de diferencia estadísticamente significativa entre aspirantes de primer y segundo año con relación a las variables actitudes y disposición al consumo de sustancias, además de identificar si existe relación entre las variables; actitudes y disposición para tener acercamientos hacia el consumo, tomando en cuenta factores de tipo psicosocial como: edad e identificación religiosa.

\section{Metodología (Materiales y métodos)}

\subsection{Hipótesis}

Las actitudes de los individuos están relacionadas con la disposición al consumo de alcohol, tabaco y otras drogas. De igual manera, consideramos que existen diferencias significativas entre aspirantes de primer y segundo año.

\subsection{Diseño}

El trabajo que se ha desarrollado comprende un estudio descriptivo y se ha utilizado la metodología cuantitativa para el análisis de poblaciones. El presente estudio integra además un análisis comparativo.

\subsection{Participantes}

Para la ejecución de la investigación, se contó con la participación de 1118 sujetos de la carrera militar que son el total de aspirantes, de los cuales, 1006 se autoidentifican como mestizos, 92 indígenas y 20 se identifican como afroecuatorianos. Sobre el estado civil, 1108 refieren ser solteros, 8 en Unión Libre y 2 comentan estar casados. Seguidamente, en la situación socioeconómica, 119 presentan una condición muy buena, 770 una condición buena, 214 una condición regular y solamente 15 una condición económica precaria. 


\subsection{Instrumentos}

Para el desarrollo se utilizó el Autoinforme de Consumo de Sustancias Psicoactivas (AICA), detallado por Macià (1986); y Santacreu, Froján y Hernández (1990): este instrumento considera siete factores: a). Conocimiento de los efectos negativos de las drogas; b). Resistencia a la idea de consumir drogas; c). Rechazo ante la invitación de tabaco y drogas ilegales; d). Rechazo incitaciones al consumo de alcohol; e). Actitud contraria al consumo de drogas normalizadas o institucionalizadas; f). Identificación entre consumo de drogas y la idea de "ser mayor"; y g). Admiración de quienes no consumen drogas normalizadas o institucionalizadas. Se ha considerado esta prueba por el alto puntaje del Alfa Cronbach de $\alpha=0,93$, evidenciando un alto índice de fiabilidad.

Consecuentemente, se utilizó el Cuestionario de actitudes hacia el alcohol y drogas ilegales (CAADI) de Macià, Méndez y Olivares (1993): consta de 30 preguntas: 10 sobre las actitudes hacia el consumo de tabaco, 10 en relación con el consumo de drogas y 10 sobre actitudes hacia el consumo de alcohol. Este cuestionario sirve para analizar la disposición personal para actuar en temas relacionados al consumo tomando en cuenta dos componentes: la reactividad y la predisposición. Además, está compuesto por tres factores para cada área (Tabaco, alcohol y otras drogas): 1. Disposición actitudinal para el consumo de tabaco, alcohol y otras drogas; 2 . Índice de desagrado o percepción de riesgo del consumo de tabaco, alcohol y otras drogas; y 3. Predisposición actitudinal para tomar acciones en contra del consumo. Hay que destacar que este cuestionario muestra una fiabilidad $\alpha=0,912$ de la aplicación en la población española.

\subsection{Procedimiento}

Posterior al contacto con autoridades de la Escuela de Formación de Soldados y después de obtener una autorización escrita, se procedió a la evaluación de los aspirantes a la carrera militar, se contó con el apoyo de 20 
psicólogos e instructores, quienes recibieron una capacitación previa sobre los instrumentos a evaluar y las posibles interrogantes que puedan surgir al momento de la evaluación.

Los aspirantes fueron evaluados de forma grupal en sus respectivos espacios y aulas, luego se elaboró una matriz en Microsoft Excel, detallando los datos de la ficha Socio Demográfica y los cuestionarios aplicados para poder exportarse al software estadístico IBM SPSS Statistics Base 22.0 y realizar el análisis de asociación de variables y el cálculo mediante tablas de contingencia.

\section{Resultados (análisis e interpretación de los resultados)}

\subsection{Análisis de asociación entre variables}

Los datos de la tabla 1, mostraron que existe una relación entre las actitudes y la disposición al consumo de tabaco. La conciencia ante los efectos negativos y mantener una actitud contraria al consumo de drogas, guarda relación con el índice de desagrado del consumo de tabaco y con la disposición de consumirlo en menor proporción.

Tabla 1. Actitudes Hacia el Tabaco (Autoinforme de Consumo de Sustancias Psicoactivas).

\begin{tabular}{|c|c|c|c|c|c|c|c|c|}
\cline { 4 - 8 } \multicolumn{2}{c|}{} & \multicolumn{7}{c|}{ Autoinforme de consumo de sustancias } \\
\cline { 3 - 10 } \multicolumn{2}{c|}{} & Fac1 & Fac2 & Fac3 & Fac4 & Fac5 & Fac6 & Fac7 \\
\hline \multirow{3}{*}{$\begin{array}{c}\text { Actitudes hacia } \\
\text { el tabaco }\end{array}$} & D.A.C.T. & 137 & 17 & 7 & 3 & 1 & 6 & 5 \\
\cline { 2 - 10 } & I.D.C.T. & 741 & 88 & 23 & 30 & 25 & 9 & 15 \\
\cline { 2 - 10 } & P.S.C.T. & 7 & 2 & 0 & 0 & 2 & 0 & 0 \\
\hline
\end{tabular}

Leyenda Factor (Fac); Disposición Actitudinal hacia el Consumo de Tabaco (D.A.C.T.); Índice de Desagrado al Consumo de Tabaco (I.D.C.T.); Percepción de Satisfacción frente al Consumo de Tabaco (P.S.C.T.).

Fuente: Los Autores (2020).

En concordancia con el análisis estadístico, en la tabla 2 se ha encontró un p-value de 0,011 lo que quiere decir que existe una asociación entre la 
actitud hacia el consumo de tabaco y los aspectos abordados en el Autoinforme de Consumo de Sustancias Psicoactivas.

Tabla 2. Chi-Square Test.

\begin{tabular}{|l|c|c|c|}
\cline { 2 - 4 } \multicolumn{1}{c|}{} & p-value & df & Asymp. Sig. (2-sided) \\
\hline Pearson Chi-Square & $26,028^{\mathrm{a}}$ & 12 &, 011 \\
\hline
\end{tabular}

Fuente: Los Autores (2020).

En cuanto a las actitudes hacia las drogas, existen 885 sujetos que revelan tener conciencia ante los efectos negativos de las drogas; valor que se relaciona con la respuesta de 762 sujetos que reflejan una identificación con la percepción de riesgo. Adicionalmente, en la tabla 3, se evidencia una relación en menor proporción en cuanto a la actitud de resistencia y la percepción de riesgo de consumo de drogas.

Tabla 3. Actitudes Hacia las Drogas (Autoinforme de consumo de sustancias Psicoactivas).

\begin{tabular}{|c|c|c|c|c|c|c|c|c|}
\cline { 4 - 4 } \multicolumn{2}{c|}{} & \multicolumn{7}{c|}{ Autoinforme de consumo de sustancias } \\
\cline { 3 - 10 } \multicolumn{2}{c|}{} & Fac1 & Fac2 & Fac3 & Fac4 & Fac5 & Fac6 & Fac7 \\
\hline \multirow{3}{*}{$\begin{array}{c}\text { Actitudes hacia } \\
\text { las drogas }\end{array}$} & D.A.C.D. & 121 & 17 & 2 & 2 & 5 & 4 & 1 \\
\cline { 2 - 10 } & P.R. & 762 & 90 & 28 & 31 & 23 & 11 & 18 \\
\cline { 2 - 10 } & P.S.C.D. & 2 & 0 & 0 & 0 & 0 & 0 & 1 \\
\hline
\end{tabular}

\begin{tabular}{|l|l|l} 
Leyenda & Disposición Actitudinal hacia el Consumo de Drogas (D.A.C.D.); Percepción de
\end{tabular} Fuente: Los Autores (2020).

En la tabla 4, posterior a la aplicación del estadístico, se pudo determinar la significancia, obteniéndose un p-value de 0,018 de asociación, dato que es menor al nivel de significación prescrito, que es de 0.05 .

Tabla 4. Chi-Square Test.

\begin{tabular}{|l|c|c|c|}
\cline { 2 - 4 } \multicolumn{1}{c|}{} & p-value & df & Asymp. Sig. (2-sided) \\
\hline Pearson Chi-Square & $24,355^{\mathrm{a}}$ & 12 &, 018 \\
\hline
\end{tabular}

Fuente: Los Autores (2020).

Se encontró además que existe una relación entre la conciencia ante 
los efectos negativos de las drogas y una actitud en contra el consumo de estas. En la tabla 5, se expone que el índice de desagrado del consumo de alcohol es significativo, lo que además se relaciona con la actitud de resistencia al consumo.

Tabla 5. Actitudes Hacia el Alcohol (Autoinforme de consumo de sustancias Psicoactivas).

\begin{tabular}{|c|c|c|c|c|c|c|c|c|}
\cline { 3 - 8 } \multicolumn{2}{c|}{} & \multicolumn{7}{c|}{ Autoinforme de consumo de sustancias } \\
\cline { 2 - 10 } \multicolumn{2}{c|}{} & Fac1 & Fac2 & Fac3 & Fac4 & Fac5 & Fac6 & Fac7 \\
\hline \multirow{3}{*}{$\begin{array}{c}\text { Actitudes hacia } \\
\text { las drogas }\end{array}$} & D.A.C.A. & 120 & 19 & 1 & 4 & 6 & 3 & 0 \\
\cline { 2 - 10 } & I.D.C.A. & 761 & 88 & 29 & 29 & 21 & 12 & 19 \\
\cline { 2 - 10 } & P.A.C.C.A. & 4 & 0 & 0 & 0 & 1 & 0 & 1 \\
\hline
\end{tabular}

\begin{tabular}{|c|l|}
\hline \multirow{2}{*}{ Leyenda } & $\begin{array}{l}\text { Disposición Actitudinal hacia el Consumo de Alcohol (D.A.C.A.); Índice de } \\
\text { Desagrado frente al Consumo de Alcohol (I.D.C.A.); Percepción Actitudinal para } \\
\text { tomar Acciones Contra el Consumo (P.A.C.C.A.). }\end{array}$ \\
\hline
\end{tabular}

Fuente: Los Autores (2020).

Se evidencia en la tabla 6 , que al realizar el análisis estadístico mediante el $X^{2}$ (Chi-Cuadrado), se determinó un p-value de 0,031 que es menor al nivel de significación del $5 \%$, concluyendo que existe asociación entre la actitud hacia el alcohol y los aspectos abordados en el Autoinforme de consumo de sustancias psicoactivas.

Tabla 6. Chi-Square Test.

\begin{tabular}{|l|c|c|c|}
\cline { 2 - 4 } \multicolumn{1}{c|}{} & p-value & df & Asymp. Sig. (2-sided) \\
\hline Pearson Chi-Square & $22,684^{\mathrm{a}}$ & 12 &, 031 \\
\hline
\end{tabular}

Fuente: Los Autores (2020).

En lo referente al análisis de diferencias estadísticamente significativa, en la tabla 7 se da a conocer la actitud de resistencia frente al consumo de drogas para los grupos analizados, encontrado que los aspirantes de segundo año tienen mayor nivel de conciencia y resistencia para consumir drogas a diferencia de aspirantes de primer año quienes tienen menor niveles de conciencia sobre los efectos negativos de consumir drogas. 
Tabla 7. Autoinforme de Consumo de Sustancias (Año del Aspirante).

\begin{tabular}{|c|l|c|c|c|}
\cline { 2 - 5 } \multicolumn{1}{c|}{} & Año Aspirante & N & Mean Rank & Test Statistic $^{\mathbf{a}}$ \\
\hline $\begin{array}{c}\text { Autoinforme de } \\
\text { Consumo de } \\
\text { Sustancias }\end{array}$ & Primero & 502 & 525,88 &, 000 \\
\cline { 2 - 5 } & Segundo & 616 & 586,90 & \\
\hline
\end{tabular}

Fuente: Los Autores (2020).

Se consideró la aplicación del estadístico de U Mann-Whitney, y se obtuvo un p-value de 0,000 por lo que se puede concluir que existen diferencias estadísticamente significativas entre los grupos estudiados con respecto al consumo de sustancias psicoactivas.

Para la tabla 8 , se visualiza que al realizar el análisis mediante el procedimiento estadístico Chi-Cuadrado, se ha obtenido un $p$-value de 0,000 lo que nos indica que existe una relación significativa entre las variables: Identificación Religiosa y los aspectos abordados en el Autoinforme de consumo de Sustancias Psicoactivas.

Tabla 8. Chi-Square Test.

\begin{tabular}{|l|c|c|c|}
\cline { 2 - 4 } \multicolumn{1}{c|}{} & p-value & df & Asymp. Sig. (2-sided) \\
\hline Pearson Chi-Square & $68,261^{\text {a }}$ & 30 &, 000 \\
\hline
\end{tabular}
Fuente: Los Autores (2020).

Se ha encontrado que la Identificación Religiosa se relaciona con el consumo de sustancias psicoactivas en sentido positivo, es decir, que tener una identificación religiosa favorece los niveles de conciencia.

\section{Conclusión}

A través del presente estudio, se encontraron diferencias por año del aspirante a la carrera militar, es así como los aspirantes de segundo año tienen mayores niveles de conciencia frente a los efectos del consumo de sustancias, en especial muestran una actitud de resistencia frente al consumo.

En los resultados encontrados se evidencia que los aspirantes de 
segundo año muestran un patrón de conducta preventivo del consumo de sustancias, manifestándose una tendencia actitudinal contraria al consumo. Igualmente, se describen diferencias en la edad: a mayor edad, los aspirantes desarrollan mayores niveles de conciencia frente a los efectos del consumo de sustancias.

En cuanto a la relación entre las actitudes frente al consumo de tabaco se encontró que los aspirantes presentan un índice de desagrado de consumo de tabaco. Se evidencia entonces que existen 137 sujetos que presentan una actitud contra el consumo de tabaco; tendencia actitudinal que aparece relacionada con la legalidad de dicha droga. Ante esto, podemos avanzar que el hecho de tener un nivel de conciencia sobre los efectos negativos del consumo no garantiza el no consumir. Los aspirantes saben que el consumo de sustancias podría causarles dificultades en el ámbito personal (deterioro de funciones), además de problemas familiares como rechazo, y bajo rendimiento en el ambiente laboral institucional.

En contraste a lo mencionado anteriormente, se hallaron 121 sujetos que denotaron una tendencia actitudinal favorable al consumo de drogas. representarían un número proporcionalmente menor. Adicionalmente, se consideró para el sujeto de análisis, ciertos datos de la ficha sociodemográfica, entre los que destaca la identificación religiosa. El hecho de tener una identificación de tipo religiosa actúa como factor protector y de desarrollo de un nivel de conciencia sobre los efectos negativos y nocivos del consumo de sustancias psicoactivas.

Finalmente se estima que el presente estudio investigativo es un valioso aporte científico para el contexto ecuatoriano y regional, ya que recoge datos de una población amplia (1118 sujetos) y obteniendo datos significativos que responden a la conciencia de efectos negativos del uso de sustancias psicoactivas y las actitudes de rechazo; información que puede ser empleada para el desarrollo de programas y planes preventivos contextualizados en 
términos socioculturales.

\section{Referencias}

Arrioja, G., Frari, S., Torres, A., Rosario, S., Avila, M., \& Morales, F. (2017). Factores de riesgo para el consumo de alcohol en adolescentes estudiantes. SMAD: Revista Eletrônica Saúde Mental Álcool E Drogas, 13(1), 22-29, e-ISSN: 1806-6976. Recuperado de:

https://doi.org/10.11606/issn.1806-6976.v13i1p22-29

Arteaga, N. (2015). Factores de riesgo asociados a los problemas relacionados con el uso de sustancias psicoactivas en población militar. Tesis. Chía, Cundinamarca, Colombia: Universidad de la Sabana; Biblioteca Octavio Arizmendi Posada. Recuperado de: https://intellectum.unisabana.edu.co/handle/10818/19243

Constitución de la República del Ecuador (2008). Sección segunda: Salud. Registro Oficial nro. 449, de fecha 20 de octubre. Ecuador: Registro Oficial; órgano de Gobierno del Ecuador. Recuperado de: https://www.registroficial.gob.ec/index.php/registro-oficialweb/publicaciones/registro-oficial/item/4864-registro-oficial-no449.html

da Silva, D., Paula, E., \& Assunção, A. (2019). Fatores associados ao uso de medicamentos ansiolíticos entre bombeiros militares. Revista Brasileira de Epidemiologia, 22, 1-14, ISSN: 1415-790X, e-ISSN: 19805497. Recuperado de:

https://doi.org/10.1590/1980-549720190021

Duarte, C., Varela, M., Salazar, I., Lema, L., \& Tamayo, J. (2012a,b). Motivaciones y recursos para el consumo de sustancias psicoactivas en universitarios. Revista Hacia la Promoción de la Salud, 17(1), 92-104, e-ISSN: 0121-7577. Recuperado de: http://www.scielo.org.co/pdf/hpsal/v17n1/v17n1a07.pdf 
Espinoza, M. (2019a,b). Factores exógenos: Incidencia en el rendimiento académico. Revista Scientific, 4(Ed. Esp.), 38-53, e-ISSN: 2542-2987. Recuperado de:

https://doi.org/10.29394/Scientific.issn.2542-2987.2019.4.E.2.38-53

Fishbein, M. (1980). A theory of reasoned action: some applications and implications. In Page, M. (Ed.): Belief, attitudes and values. Lincoln, United States: University of Nebraska Press.

Fuentes, E., Litardo, F., \& Castro, W. (2017). Las Funciones y Atribuciones de la Secretaría Técnica de Drogas (SETED) Ecuador. RECIMUNDO: Revista Científica Mundo de la Investigación y el Conocimiento, 1(5), 303-334. Recuperado de:

https://doi.org/10.26820/recimundo/1.5.2017.303-334

Gribble, R., Goodwin, L., \& Fear, N. (2019). Resultados de salud mental y consumo de alcohol entre esposas/parejas de militares del Reino Unido: una comparación con las mujeres en la población general. European Journal of Psychotraumatology, 10(1), 1-13, e-ISSN: 20008198. Recuperado de: https://doi.org/10.1080/20008198.2019.1654781 Macià, D. (1986). Cuestionario de Actitudes acerca del Alcohol y Cuestionario de información. Método Conductual de Prevención de Drogodependencias. Valencia, España: Editorial Promolibro.

Macià, D., Méndez, F., \& Olivares, J. (1993). Intervención psicológica: Programas aplicados de tratamiento. Madrid, España: Ediciones Pirámide.

Meadows, S., Engel, C., Collins, R., Beckman, R., Cefalu, M., Hawes-Dawson, J., .. Williams, K. (2018a,b). 2015 Health Releated Behaviors Survey: Deployment Experiences and Health Among U.S. Active-Duty Service Members. Santa Monica, C.A., United States: RAND Corporation. Recovered from: https://doi.org/10.7249/RB9955.1

Mendoza, Y., \& Vargas, K. (2017). Factores psicosociales asociados al 
consumo y adicción a sustancias psicoactivas. Revista electrónica de psicología de Iztacala, 20(1), 139-167, e-ISSN: 1870-8420. Recuperado de:

http://www.revistas.unam.mx/index.php/repi/article/view/58927

Meneses, K., Cisneros, M., \& Braganza, M. (2019). Análisis socioeconómico del consumo excesivo de alcohol en Ecuador. Revista Ciencias de la Salud, 17(2), 293-308, e-ISSN: 2145-4507. Recuperado de: http://dx.doi.org/10.12804/revistas.urosario.edu.co/revsalud/a.7938

Moreta-Herrera, R., Ilaja-Verdesoto, B., Mayorga-Lascano, M., León-Tamayo, \& López-Castro, J. (2018a,b). Actitudes y disposición personal ante el consumo y exposición a sustancias en adolescentes del Ecuador. Salud y Drogas, 18(2), 217-226, e-ISSN: 1988-205X. Recuperado de: https://doi.org/10.21134/haaj.v18i2.383

National Institute on Drug Abuse (2019). El consumo de drogas y la vida militar. DrugFacts. United States: Department of Health \& Human Services-USA. Recuperado de:

https://www.drugabuse.gov/sites/default/files/drugfacts-abuse-militarysp.pdf

OPS (2018). Informe sobre el control del tabaco en la Región de las Américas. ISBN: 978-92-75-32015-0. Washington, D.C., Estados Unidos: Organización Panamericana de la Salud.

Organización de los Estados Americanos (2019a,b,c,d). Informe sobre el consumo de Drogas en las Américas. ISBN: 978-0-8270-6794-3. Washington, D.C.: Comisión Interamericana para el Control del Abuso de Drogas (CICAD), Organización de los Estados Americanos (OEA).

Ruisoto, P., Cacho, R., López-Goñi, J., Vaca, S., \& Jiménez, M. (2016). Prevalencia y perfil de consumo de alcohol en estudiantes universitarios en Ecuador. Gaceta Sanitaria, 30(5), 370-374, e-ISSN: 0213-9111. Recuperado de: 
https://doi.org/10.1016/j.gaceta.2016.02.008

Santacreu, J., Froján, M., \& Hernández, J. (1990). AICA: Autoinforme sobre

Consumo de sustancias psicoactivas. Departamento de Psicología de la Salud. España: Universidad Autónoma de Madrid.

Santrock, J. (2002). Psicología de la educación. México: McGraw-Hill.

Vargas, C., Castellano, E., \& Trujillo, H. (2017a,b). Factores asociados al consumo de drogas en una muestra de militares españoles desplegados en "Bosnia-Herzegovina". Adiciones, 29(3), 163-171, eISSN: 0214-4840. Recuperado de:

https://doi.org/10.20882/adicciones.734

Vera, J., Quintana, J., Valdés, Á., Martínez, L., \& Martínez, E. (2014). Model for measuring teenagers' alcohol consumption intent. Interamerican Journal of Psychology, 48(2), 327-339, e-ISSN: 2329-4795. Recuperado de:

https://journal.sipsych.org/index.php//JP/article/view/270

Vélez, R., \& Gudiño, D. (2017). Política de drogas en Ecuador: Un balance cuantitativo para transformaciones cualitativas. Quito, Ecuador: Friedrich-Ebert-Stiftung (FES-ILDIS). Recuperado de: https://www.fesecuador.org/news-list/e/politica-de-drogas-en-ecuador-un-balancecuantitativo-para-transformaciones-cualitativas/

World Health Organization (2014). Global status report on alcohol and health. ISBN: 978-92-4-156475-5, ISBN: 978-92-4-069276-3. Switzerland: World Health Organization. 


\section{Joel Santiago Barreno López}

e-mail: jsbarreno.mpp@uisek.edu.ec

Nacido en Ambato, Ecuador, el 25 de septiembre del año

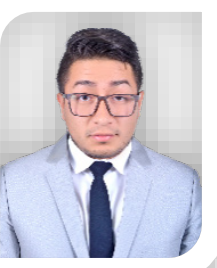

1990. Psicólogo clínico; Máster en Psicología con mención en Psicoterapia por la Universidad Internacional SEK (UISEK); Experiencia clínica en Comunidades Terapéuticas para pacientes drogodependientes; Fundador del grupo Terapeutico Recuper-Arte. 


\section{Claudio Danilo Pazmiño Tirado}

e-mail: cdpazmino.mpp@uisek.edu.ec

Nacido en Pelileo, Ecuador, el 8 de noviembre del año

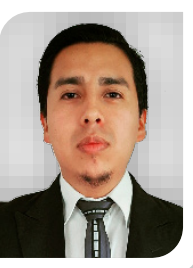
1989. Psicólogo clínico; Máster en Psicología con mención en Psicoterapia por la Universidad Internacional SEK (UISEK); Experiencia clínica en Centros de Recuperación para pacientes drogodependientes; Psicólogo del grupo Terapéutico Recuper-Arte. 
DOI: https://doi.org/10.29394/Scientific.issn.2542-2987.2020.5.E.10.205-225

OAI-PMH: http://www.indteca.com/ojs/index.php/Revista_Scientific/oai

Artículo Original / Original Article

\section{Luis Alfonso Iriarte Pérez}

e-mail: luisiriartep@gmail.com

Nacido en Maracaibo, Venezuela, el 13 de octubre del año

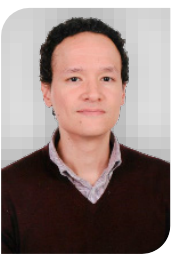
1985. Dr. Psicología (PhD.); Máster en Ciencias Sociales y Magister Scientiarum en Psicología Clínica; Profesor a tiempo parcial en la Universidad San Francisco de Quito (USFQ). 